\title{
Parálisis periódica hipopotasemia
}

\section{Hypokalemic periodic paralysis}

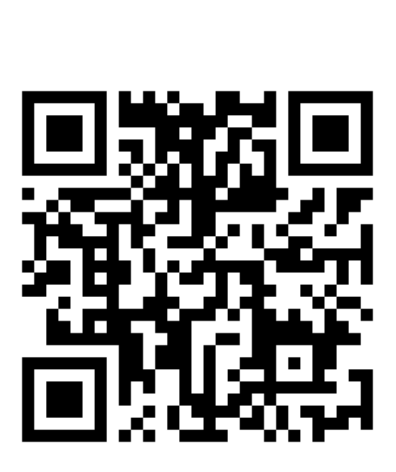

Recibido 22/06/2021
Corregido $30 / 06 / 2021$
${ }^{1}$ Dra. Katherine Campos Duarte Hospital Nacional Psiquiátrico, San José, Costa Rica

(iD) https://orcid.org/0000-0002-0195-1110

2Dra. Mónica Mesén Román Hospital Nacional Psiquiátrico, San José, Costa Rica (iD) https://orcid.org/0000-0002-9927-1112

${ }^{3}$ Dra. Astrid Cartín Ramírez Autism Awareness Costa Rica, Costa Rica

(iD) https://orcid.org/0000-0001-5963-8605

\section{RESUMEN}

La parálisis periódica hipocalemia es un trastorno autosómico dominante neuromuscular, que forma parte de un conjunto de enfermedades agrupadas conocidas como canalopatías. Estas diversas patologías se caracterizan por presentar mutaciones en los genes ubicados en los canales iónicos de sodio y calcio dependientes del voltaje, los cuales generan una inexitabilidad de la membrana muscular. Presentan diversas manifestaciones clínicas como episodios de parálisis y debilidad muscular de los cuatro miembros que puede durar horas, no obstante, suelen afectar principalmente a nivel de los miembros inferiores, relacionados con niveles bajos de potasio en sangre. Se describen dos genes causantes de las alteraciones en el voltaje denominados CACNA1S, SCN4A.

El diagnóstico se basa en la historia clínica y sintomatología, así como la confirmación mediante pruebas genéticas, la adecuada educación acerca de la identificación de los factores de riesgo, consejos dietéticos, cambios estilo de vida junto con intervenciones farmacológicas son el tratamiento de elección ideal.

PALABRAS CLAVE: hipocalémica; canalopatías; debilidad muscular; parálisis.

\section{ABSTRACT}

The hypokalemic paralysis is a dominant neuromuscular autosomal disorder, that belongs to a group of diseases known as channelopathies. These kinds of diseases are characterized for showing mutations on the genes located on the ionic channels of sodium and calcium defendant on the voltage, which cause an inexatibility of the muscular membrane. They present several 
clinical manifestations like episodes of paralysis and muscle weakness of the 4 body members which can last hours, nonetheless it usually affects the lower members of the body, related with low levels of potassium on the blood. There are two genes which cause the alterations in the voltage denominated CACNA1S, SCN4A.

The diagnosis is based on the clinical history as well the symptomatology, as well confirmation through genetic tests, the adequate education about identifying risk factors, dietary advice, lifestyle changes and well pharmacological intervention are the ideal treatment for the condition.

KEYWORDS: hypokalemic; channelopathies; muscular weakness; paralysis.

${ }^{1}$ Médica general, graduada de la Universidad de Hispanoamericana (UH). Cód. MED16688. Correo: katy002002@gmail.com

${ }^{2}$ Médica general, graduada de la Universidad de Hispanoamericana (UH). Cód. MED15011. Correo: mesenmonica@gmail.com

${ }^{3}$ Médica general, graduada de la Universidad de Ciencias Médicas (UCIMED). Cód. MED16085. Correo: dracartinr@gmail.com

\section{INTRODUCCIÓN}

Las canalopatías musculares se definen como un conjunto de enfermedades de origen genético, poco frecuentes $y$ heterogéneos, existen múltiples mutaciones a nivel de los canales iónicos dependientes del voltaje, estas alteraciones en estos canales. Ocasionan modificaciones específicamente en el ciclo de la excitabilidad en membrana celular dando como resulto una pérdida de función representada en parálisis $(1,2,3)$.

Se clasifican en tres distintos grupos de patologías las miotonias no distróficas, parálisis periódicas (PP) y la hipertermia maligna. A su vez las PP se dividen en varios subgrupos estas se llaman parálisis periódica hipopotasemia (HypoPP), parálisis periódica hiperpotasemia (HyperPP) y síndrome de Andersen-Tawil (ATS) $(3,4,5)$. La HypoPP puede ocurrir y dar sintomatología bajo tres panoramas. De origen primerio sea genético, tirotoxicosis o por causas secundarias que provoquen hipopotasemia por otros mecanismos asociado a pérdida de potasio gastrointestinal (3).

Dentro de su etiología genética, se encuentra como causas la mutación en genes que codifican y se encuentran ubicados en los canales de calcio CACNA1S, gen que codifica la subunidad alfa-1 en este canal el cual es sensible a la dihidropiridina ubicado en el músculo esquelético, es la forma más común y representa aproximadamente $60-70 \%$ de los casos, a su vez en menor frecuencia existe una alteración en los canales de sodio en el músculo esquelético llamado SCN4A, representa una menor incidencia entre el 10$20 \%$ aproximadamente, ambos se encuentran ubicados en la membrana citoplasmática del músculo esquelético o sarcolema, los cuales son esenciales para diversas funciones como es el acoplamiento de excitación-contracción y la excitabilidad sarcolémica $(6,7)$.

Si bien no está descrito certeramente como funciona la inactivación de estos canales, se cree que estas mutaciones a nivel de los diferentes canales de calcio y sodio ocasionan un defecto en la pérdida de función, así como una activación o bien inactivación más lenta de estos canales. Que a su vez dan como resultado una despolarización parcial de la membrana que genera inexcitabilidad de las fibras musculares por inactivación de los mismos canales (2).

Tanto las mutaciones en los canales de calcio o sodio pueden causar PP del mismo tipo ya sea HypoPP o ATS. Sin embargo, el gen que codifica el canal de sodio puede causar distintas mutaciones y en 
consecuencia causar PP (hipopotasemia, normokalaémica o hiperpotasémica) $(1,4,8)$. Su presentación clínica no varía y es idéntica en los pacientes, ya sea causado por mutaciones del canal del calcio o sodio. Ambas alteraciones ocasionan cambios que afectan la permeabilidad de la membrana celular, generando salida anómala de potasio que a su vez afecta el potencial de acción, provocando inestabilidad en la despolarización paradójica de la fibra y a la inexcitabilidad en el ajuste de $\mathrm{K}$ extracelular bajo $(9,10)$.

Si bien no es una patología común, es por este medio que se busca ampliar el conocimiento y dar a conocer con mayor detalle esta patología mediante esta revisión, en donde se realiza una diversa búsqueda bibliográfica que permite sintetizar y recopilar las principales manifestaciones clínicas, fisiopatología, diagnóstico y tratamiento.

\section{MÉTODO}

Para esta publicación se usaron buscadores como, SCIELO, Access medicine, Cochrane Library, Pubmed, Elsevier, Clinical Key, Science Direct se utilizaron la combinación de descriptores como: "parálisis periódica", "canalopatías", "Hypokalemic periodic paralysis", "THPP ", "HypoKPP" para la misma. Se seleccionaron artículos tanto en inglés como español. Toda la bibliografía utilizada fue publicada en un período no mayor a los cinco años del año actual.

Dicha búsqueda se enfocó fundamentalmente, en aquellos artículos en el cual se logró revisar y analizar el texto completo, con la mayor relevancia y mejor calidad de información, se descartó todos aquellos artículos que se consideraron con deficiencias en la metodológica importantes, información desactualizada, los que se abordó poco, deficiente o no de manera correcta el tema en específico, Adicionalmente se consultaron libros de referencia como Principios de Medicina Interna, Principios de neurología, Neurología clínica y neuroanatomía. Un enfoque basado en la localización.

\section{HISTORIA}

Las primeras descripciones acerca de la HypoPP, ocurrió durante los años 1800, mediante la observación por parte de los científicos Hartwig (1874), Westphal (1885), Oppenheim (1891), así como de Goldflam (en 1895) en donde detallaron los primeros hallazgos con respecto a la vacuolización del sarcoplasma que está presente en las fibras musculares característica de esta patología, por otra parte Aitken et en el año 1937, relaciona las concentraciones de potasio disminuidas séricas durante los ataques de parálisis, a su vez explica que esta condición descrita puede revertir con la administración de potasio, para el año 1991-1994 se realiza las identificaciones de las primeras mutaciones presentes en la PP ubicado en el gen SCN4A 1991 y CACNA1S en 1994 por parte de Ptacek et al. $(11,12)$.

\section{GENERALIDADES}

Se define como un trastorno autosómico dominante es decir se requiere únicamente una copia genética anormal para generar manifestaciones clínicas, es la patología más frecuente dentro del grupo de las PP, presenta una prevalencia aproximada de 1 en cada 100.000 habitantes, se presenta con mayor frecuencia en hombres que en mujeres, con inicio predominantemente en la adolescencia $(3,4,13)$.

Presenta debilidad de características episódicas y parálisis muscular los cuales se ven asociados con niveles bajos de potasio menores de $3 \mathrm{mEq} / \mathrm{l}(4)$.

Estos niveles bajos de potasio se pueden deber a dos causas que son de origen primario de causa tirotóxica y la HypoPP 
familiar o secundarias asociado a pérdida de potasio gastrointestinal (14).

Dentro de cambios patológicos se pueden observar como las fibras musculares que se encuentran de manera uniforme son un poco más grandes de los usual, pueden experimentar degeneración segmentaria, a su vez en etapas más avanzadas y degenerativas de la enfermedad, se observa vacuolización de aspecto redondas u ovales del sarcoplasma en donde se observan cambios patológicos en las miofibrillas y las mitocondrias $(12,15)$.

\section{PRESENTACIÓN CLÍNICA}

Se desarrolla en la segunda década de la vida, siendo menos frecuentes después de los 40 años. Posee características distintivas, como son los episodios recurrentes, reversibles de debilidad muscular flácida, bilateral y simétrica. Puede agravarse y progresar a una debilidad muscular permanente, generalmente se presentan por la noche o temprano en la mañana. Ocurren de manera espontánea o bien asociado con un deterioro en la calidad de vida debido a la fatiga, debilidad muscular, son predominantemente nocturnos o temprano en la mañana $(3,16)$. A su vez existen síntomas pródromos previo a la debilidad y parálisis los pueden estar presentes como el hambre, sed en exceso, xerostomía, palpitaciones, sudoración, diarrea, nerviosismo y sensación de laxitud o fatiga (12).

Se desarrolla en diversos grados de debilidad entre ataques, así mismo un grupo amplio de pacientes afectados desarrollaran debilidad persistente más adelante en la vida (10).

Dentro los factores desencadenantes se representan por parálisis flácida episódica que suele ser en respuesta a diversas causas como puede ser el reposo después de ejercicio agotador, frío o factores de la dieta como comidas ricas en carbohidratos favorecen su aparición $(10,16)$.

Tiene como característica presentar inicialmente disminución del tono muscular, mayoritariamente en extremidades que suelen verse afectadas con mayor gravedad que los músculos del tronco, tiende a ser más marcada proximalmente que distalmente, así como las piernas más que a los brazos, además cuando la crisis se encuentra en su fase máxima, los reflejos tendinosos y reflejos cutáneos pueden verse afectados de tal manera de reducirse o desaparecer por lo que la hiporreflexia o arreflexia es un patrón típico (17).

Episodios más graves también pueden verse comprometidos como la musculatura ocular, bulbar y respiratoria, así como la deglución. Sin embargo, se describen variantes atípicas de estos episodios donde pueden desarrollar a lo largo del tiempo debilidad de forma permanente con mayor predominio en los miembros inferiores o afectar la musculatura ocular, respiratoria, deglución entre otros $(4,12)$.

Se puede presentar por intervalos de tiempos que pueden variar de semanas a meses, su sintomatología se caracteriza por ser de corta duración que puede varias de minutos a horas, no obstante, puede necesitar hasta un periodo de 24 horas, para mostrar resolución completa de los síntomas $(18,14)$.

Esta descrito que en casos severos de HypoPP pueden presentar arritmias cardíacas, que a su vez el descenso de potasio presenta manifestaciones en el trazo del electrocardiograma (ECG), hallazgos como depresión del segmento ST, disminución en la amplitud de la onda T y un aumento en la amplitud de las ondas $U$; no obstante, el corazón y los músculos lisos no se ven afectados por la enfermedad (13).

\section{DIAGNÓSTICO}


El diagnóstico se estable mediante la historia clínica, pruebas electromiografías además de pruebas genéticas, asociados con potasio sérico disminuidos y documentados menores a $<3,5 \mathrm{mmol} / \mathrm{L}$ durante los ataques, a su vez se relacionan otros tipos de electrolitos afectados, como la hipofosfatemia e hipomagnesemia leves y enzimas elevadas en casos graves tales como creatinfosfoquinasa, enzima aspartato aminotransferasa y lactato deshidrogenasa $(3,18)$.

Inicialmente se debe sospechar con base a la historia clínica y anamnesis minuciosa que permita una mejor orientación diagnóstica, en donde se debe sospechar en individuos que se describan ataques episódicos y recurrentes de parálisis flácida o debilidad, así de historia familiares de parálisis episodicas $(4,13)$.

A su vez la identificación de una variante patogénica heterocigótica puede ser confirmado mediante una prueba genética entre $60 \%$ al $70 \%$ de los pacientes que cumplen los criterios clínicos en el gen es CACNA1S o SCN4A y el cromosoma es 1q31-32 o 17q23-25; no obstante, se estima que aproximadamente el $30 \%$ pueden no tener una variante patógena identificada en ninguno de estos genes descritos previamente conocidos (10).

El ejercicio físico un factor desencadenante, que puede ser agravante $o$ atenuante de los síntomas, es por ello y basado en este principio el ejercicio puede ser utilizado dentro de su diagnóstico como una prueba funcional en electromiografía, mediante la prueba de provocación con ejercicio (1).

Se ha evidenciado que los estudios electrofisiológicos no son de gran utilidad en crisis agudas para su diagnóstico, debido a que se debe realizar posterior a la crisis; sin embargo, se puede observar la ausencia de descargas miotónicas, presencia de progresiva, así como de una marcada disminución en la amplitud de los potenciales de acción motora, así como una reducción de la velocidad de conducción de las fibras musculares (14).

Dentro de los hallazgos histológicos que podemos encontrar en una biopsia muscular se puede observar vacuolas centrales en las fibras musculare, con depósitos de glucógeno dentro de ellas que alteraran la estructura de la fibra muscular, lo que finalmente podría estar relacionado con las fibras musculares distróficas (15).

\section{TRATAMIENTO}

Es importante individualizar en cada paciente, el manejo consiste en tratamiento preventivo con cambios en el estilo de vida y evitar los desencadenantes los cuales son suficientes en cierto grupo de pacientes, mientras que otro grupo de individuos se benefician de intervenciones farmacológicas adicional a estos cambios $(2,10)$.

Las cuales consisten en abortar los ataques agudos y de forma preventiva manejo crónico para reducir la frecuencia de los ataques, con el fin de evitar o disminuir a largo plazo evitar las crisis $(10,13)$.

\section{- Tratamiento agudo}

Dentro del tratamiento farmacológico agudo varia en relación con la gravedad y duración de los síntomas. Usualmente los ataques leves tienden a resolver espontáneamente, el objetivo principal consiste en prevenir las futuras complicaciones como arritmias $(3,19)$.

Para las crisis agudas leves, se puede administrar por vía oral una dosis de 0,5 a $1 \mathrm{mEq} / \mathrm{kg}$ hasta $200 \mathrm{mEq} / 24$ horas hasta la normalización, existen ciertas condiciones en donde los paciente se benefician de tratamiento intravenoso, en casos como cuando no se tolera la vía oral sea por problemas de deglución, vómitos o en escenarios de crisis agudas graves, en donde no hay mejoría significativa al pasar 1 a 2 horas, es por ello que en estos casos la administración de $\mathrm{KCl}$ por vía intravenosa a $40 \mathrm{mEq} / \mathrm{l}$ 
en solución de manitol al $5 \%$, sin exceder de $20 \mathrm{mEq} / \mathrm{hora}$ ni $200 \mathrm{mEq} /$ día es el tratamiento idóneo, no se debe administrarse sueros glucosados 0 solución salina, debido a que pueden ocasionar un aumento de insulina endógena que agravará la hipopotasemia promoviendo aún más la entrada de potasio en el musculo esquelético $(2,4,13)$.

\section{- Tratamiento preventivo}

Las medidas preventivas no farmacológicas consisten en la educación acerca de dieta y estilo de vida, como lo son tener una dieta hiposódica (menos de $160 \mathrm{mEq} /$ día), dieta baja en carbohidratos, ingestas menos copiosas, pero con mayor frecuencia, evitar la exposición al frío y el ejercicio intenso, son algunos de estos factores a evitar $(2,13)$.

- Tratamiento crónico a largo plazo

A su vez acetazolamida 250 mg cada 12 horas, sirve para prevenir futuras crisis, el cual puede ser administrado de manera profiláctica, en aquellos pacientes que presentan mutaciones en los canales CACNA1S son los que mejor responden al tratamiento, sin embargo para pacientes con mutaciones en SCN4A, estos suelen ser refractarios a tratamiento e incluso pueden presentar una exacerbación de los síntomas, es por ello que se benefician con el diclorfenamida (50 a $150 \mathrm{mg} / \mathrm{día})$, espironolactona o triamtereno $(4,12)$.

\section{CONCLUSIONES}

La HypoPP, forma parte de las enfermedades que conforman el grupo de las canalopatías musculares de origen genético, su clínica puede llegar a afectar la calidad de vida debido a sus episodios de debilidad muscular flácida, bilateral y simétrica, su duración es variable de horas a días. Inicia generalmente durante la adolescencia, los hombres suelen verse afectados con mayor frecuencia que las mujeres, su sintomatología se encuentra asociado con la disminución de los niveles de potasio en sangre.

Su clínica se presenta principalmente a nivel de las extremidades, respetando musculatura facial y respiratoria, sin embargo, en casos graves no se exime de que puedan verse afectados. Su sintomatología suele ser intermitente y reversible, sin embargo, puede progresar ha debilidad muscular permanente y progresiva principalmente en músculos proximales de las extremidades inferiores. Factores como el ejercicio físico, dieta rica en carbohidratos, exposición al frio entre otros, estas descritos como causas desencadenantes para desarrollar sintomatología.

El tratamiento consiste en tres pilares importantes que involucra el tratamiento farmacológico para las crisis agudas mediante la administración de KCL sea por vía oral o intravenosa, tratamiento crónico a largo plazo preventivo y medidas no farmacológicas como la educación acerca de evitar los factores desencadenantes, dieta y estilo de vida.

\section{Los autores declaran no tener conflicto de interés.}

\section{REFERENCIAS}

1. Berardo A, lanardi S, Wilhelm C, Reisin R. Caracterización clínica, electrofisiológica y molecular en un paciente con parálisis periódica hipocalémica tipo 2, nueva mutación en SCN4A. Neurol Argent. 1 de octubre de 2016;8(4):277-82.

2. Jitpimolmard N, Matthews E, Fialho D. Treatment Updates for Neuromuscular Channelopathies. Curr Treat Options Neurol [Internet]. 2020 [citado 16 de junio de 2021];22(10). Disponible en: https://www.ncbi.nlm.nih.gov/pmc/articles/PMC74 43183/

3. Ha D, Pacd P. Periodic paralysis: what clinician needs to know? Endocrinol Int J [Internet]. 18 de julio de 2018 [citado 20 de junio de 2021];6(4). Disponible en: https://medcraveonline.com/EMIJ/periodicparalysis-what-clinician-needs-to-know.html 
4. Zapata-Wainberg G, Sacristana MG de la, Vivancos J. Canalopatías del músculo esquelético de base genética: parálisis periódicas y miotonías no distróficas. Med - Programa Form Médica Contin Acreditado. abril de 2019;12(76):4478-85.

5. Ortega M, Marcela M. Parálisis periódica hipopotasémica familiar, Instituto Hondureño de Seguridad Social. Rev Fac Cienc Méd Impr. 2019;52-61.

6. Latorre R, Purroy F. Parálisis periódica hipocaliémica: revisión sistemática de casos publicados. Rev Neurol. 2020;71(09):317.

7. Sampedro Castañeda M, Zanoteli E, Scalco RS, Scaramuzzi V, Marques Caldas V, Conti Reed U, et al. A novel ATP1A2 mutation in a patient with hypokalaemic periodic paralysis and CNS symptoms. Brain. 1 de diciembre de 2018;141(12):3308-18.

8. Zurawski M, Severini M, Malfante P. PARALISIS PERIODICA HIPOCALEMICA TIROTOXICA. PRESENTACIÓN DE CUATRO CASOS. 2016;4.

9. Reyes-Toledo R, Culma-Roa L, Medinan-Mur R. Parálisis periódica hipocalémica: reporte de caso. Rev Ecuat Neurol. 2020;29(3):97-101.

10. Statland JM, Fontaine B, Hanna MG, Johnson NE, Kissel JT, Sansone VA, et al. Review of the Diagnosis and Treatment of Periodic Paralysis. Muscle Nerve. abril de 2018;57(4):522-30.

11. Fialho D, Griggs RC, Matthews E. Periodic paralysis. En: Handbook of Clinical Neurology [Internet]. Elsevier; 2018 [citado 15 de junio de 2021]. p. 505-20. Disponible en: https://linkinghub.elsevier.com/retrieve/pii/B9780 444640765000326

12. Ropper AH, Samuels MA, Klein JP, Prasad S. Trastornos de la unión neuromuscular, miotonías y actividad persistente de las fibras musculares. En: Adams y Victor Principios de neurología [Internet]. 11e ed. New York, NY: McGraw-Hill Education; 2019 [citado 16 de junio de 2021]. Disponible en: accessmedicina.mhmedical.com/content.aspx?ai $\mathrm{d}=1174939390$

13. Amato AA, Brown Jr Robert $H$. Distrofias musculares y otras enfermedades del músculo. En: Jameson JL, Fauci AS, Kasper DL, Hauser SL, Longo DL, Loscalzo J, editores. Harrison Principios de Medicina Interna, 20e [Internet]. New York, NY: McGraw-Hill Education; 2018 [citado 15 de junio de 2021]. Disponible en: accessmedicina.mhmedical.com/content.aspx?ai $\underline{\mathrm{d}=1174534515}$

14. Rojas W, Flórez A, Pinzón D. Parálisis periódica hipocalémica: reporte de caso y revisión del tema. Repert Med Cir. enero de 2017;26(1):35-8.

15. Holm-Yildiz S, Krag T, Witting N, Duno $M$, Soerensen T, Vissing J. Vacuoles, Often Containing Glycogen, Are a Consistent Finding in
Hypokalemic Periodic Paralysis. J Neuropathol Exp Neurol. 1 de octubre de 2020;79(10):1127-9.

16. Berkowitz AL. Enfermedades del músculo. En: Neurología clínica y neuroanatomía Un enfoque basado en la localización [Internet]. 1ra ed. New York, NY: McGraw-Hill Education; 2020 [citado 10 de junio de 2021]. Disponible en: accessmedicina.mhmedical.com/content.aspx?ai $\mathrm{d}=1175410998$

17. Holm-Yildiz S, Witting N, Dahlqvist J, de Stricker Borch J, Solheim T, Fornander F, et al. Permanent muscle weakness in hypokalemic periodic paralysis. Neurology. 28 de julio de 2020;95(4):e342-52.

18. Weber F, Lehmann-Horn F. Hypokalemic Periodic Paralysis. En: Adam MP, Ardinger HH, Pagon RA, Wallace SE, Bean LJ, Mirzaa G, et al., editores. GeneReviews ${ }^{\circledR} \quad$ [Internet]. Seattle (WA): University of Washington, Seattle; 2018 [citado 16 de junio de 2021]. Disponible en: http://www.ncbi.nlm.nih.gov/books/NBK1338/

19. Sardar Z, Waheed KhAF, Javed MA, Akhtar F, Bokhari SRA. Clinical and Etiological Spectrum of Hypokalemic Periodic Paralysis in a Tertiary Care Hospital in Pakistan. Cureus [Internet]. 19 de enero de 2019 [citado 16 de junio de 2021]; Disponible en: https://www.cureus.com/articles/10642-clinicaland-etiological-spectrum-of-hypokalemicperiodic-paralysis-in-a-tertiary-care-hospital-inpakistan 